\title{
Effects of confinement and external fields on structure and transport in colloidal dispersions in reduced dimensionality
}

\author{
D Wilms ${ }^{1,2}$, S Deutschländer ${ }^{3}$, U Siems $^{3}$, K Franzrahe $^{3}$, P Henseler ${ }^{3}$, \\ P Keim ${ }^{3}$, N Schwierz ${ }^{3}$, P Virnau ${ }^{1}$, K Binder ${ }^{1}$, G Maret $^{3}$ and P Nielaba $^{3}$ \\ ${ }^{1}$ Institut für Physik, Johannes Gutenberg-Universität Mainz, Staudinger Weg 7, D-55099 Mainz, \\ Germany \\ ${ }^{2}$ Graduate School Materials Science in Mainz, Staudinger Weg 9, D-55128 Mainz, Germany \\ ${ }^{3}$ Physics Department, University of Konstanz, D-78457 Konstanz, Germany \\ E-mail: wilmsdo@uni-mainz.de
}

\begin{abstract}
In this work, we focus on low-dimensional colloidal model systems, via simulation studies and also some complementary experiments, in order to elucidate the interplay between phase behavior, geometric structures and transport properties. In particular, we try to investigate the (nonlinear!) response of these very soft colloidal systems to various perturbations: uniform and uniaxial pressure, laser fields, shear due to moving boundaries and randomly quenched disorder.

We study ordering phenomena on surfaces or in monolayers by Monte Carlo computer simulations of binary hard-disk mixtures, the influence of a substrate being modeled by an external potential. Weak external fields allow a controlled tuning of the miscibility of the mixture. We discuss the laser induced de-mixing for the three different possible couplings to the external potential.

The structural behavior of hard spheres interacting with repulsive screened Coulomb or dipolar interaction in 2D and 3D narrow constrictions is investigated using Brownian dynamics simulations. Due to misfits between multiples of the lattice parameter and the channel widths, a variety of ordered and disordered lattice structures have been observed. The resulting local lattice structures and defect probabilities are studied for various cross sections. The influence of a self-organized order within the system is reflected in the velocity of the particles and their diffusive behavior.

Additionally, in an experimental system of dipolar colloidal particles confined by gravity on a solid substrate we investigate the effect of pinning on the dynamics of a two-dimensional colloidal liquid.

This work contains sections reviewing previous work by the authors as well as new, unpublished results. Among the latter are detailed studies of the phase boundaries of the de-mixing regime in binary systems in external light fields, configurations for shear induced effects at structured walls, studies on the effect of confinement on the structures and defect densities in three-dimensional systems, the effect of confinement and barriers on two-dimensional flow and diffusion, and the effect of pinning sites on the diffusion.
\end{abstract}

\section{Introduction}

The physics of surfaces and adsorbed monolayers has attracted a lot of interest in the context of soft matter research and its promising, versatile technical applicability. In theoretical studies two-dimensional systems are often used as models, the interactions with substrates being conveniently modeled by external fields. The experimental counterpart, 
two-dimensional systems of colloidal suspensions, has been studied extensively in recent decades. A close interplay between experiments, analytic theory and computer simulations helped to shed light on such fundamental questions of statistical physics as the nature of melting in two dimensions with and without the influence of external fields. In this context the elastic properties of two-dimensional colloidal crystals have been analyzed by us recently $[1,2]$, as well as the effect of external fields on structures and phase transitions [3-7].

This work contains parts reviewing previous work by the authors as well as new, unpublished results. Among the latter are detailed studies of the phase boundaries of the de-mixing regime in binary systems in external light fields, configurations for shear induced effects at structured walls, studies on the effect of confinement on certain structures and defect densities in three-dimensional systems, the effect of confinement and barriers on two-dimensional flow and diffusion, and the effect of pinning sites on the diffusion.

In this work we focus on low-dimensional colloidal model systems, via simulation studies and also some complementary experiments, in order to elucidate the interplay between phase behavior, geometric structures and transport properties. In particular, we try to investigate the (nonlinear!) response of these very soft colloidal systems to various perturbations: uniform and uniaxial pressure, laser fields, shear due to moving boundaries and randomly quenched disorder.

The majority of our studies has been carried out in two-dimensional systems. The search for methods to induce certain structures in colloidal systems has fostered the investigation of the effects of external light fields and of structured (one-dimensional) walls on the structure of model colloids. In this context sections 2 and 3 show new contributions. Section 2 concentrates on the interesting effect of laser induced de-mixing [3]. Here a detailed study of the boundaries of the de-mixing regime within the phase diagram is presented. Section 3 focuses on shear induced structural deformations near walls. The effect of the confinement on the dynamics of such systems is studied in greater detail in section 3 as well as in section 4, where particles are exposed to external fields in addition to the wall confinement. The first particle layers close to the walls are of particular interest in section 4, where interesting layering effects have been found for certain well chosen wall distances, resulting in a small number of layers. In section 3 complementary studies have been carried out with the focus on larger layer numbers and the effect of shear on the ordering in the first layers close to the confining walls. In sections 4 and 5 finally the diffusion of particles has been analyzed in greater detail. In section 5 pinning sites result in a subdiffusive behavior, and in section 4 the formation of layers hinders the free diffusion of the particles resulting in a subdiffusive behavior as well. In both systems, this behavior is only found at intermediate timescales.

The paper is organized as follows. In section 2 we present the results of Monte Carlo studies on laser induced de-mixing as observed in binary hard-disk mixtures. In section 3 we show Monte Carlo simulation results of the effect of one-dimensional confining corrugated walls on phase transitions of two-dimensional colloidal crystals and on their behavior under shear. Section 4 presents our results of Brownian dynamics simulation studies of the transport of colloidal systems in two- and three-dimensional channels, and in section 5 experimental results of the pinning effect on the dynamics of a two-dimensional colloidal liquid are presented.

\section{Binary two-dimensional model colloids in external fields}

Monodisperse 2D colloidal systems in interaction with a substrate potential have been studied extensively in experiments [8, 9], computer simulations [10, 6, 11, 5] and theory [12-14] over recent decades. Reentrant phase transition scenarios like laser induced freezing (LIF) and laser induced melting (LIM) have been observed. In recent studies $[3,4]$ we addressed the question of how the addition of another length scale into such a system influences the intricate competition between adsorbate-adsorbate and adsorbate-substrate interaction by studying a binary $50 \%$ mixture under the influence of a 1D spatially periodic substrate potential. The colloids are modeled by hard disks with a diameter ratio $\sigma_{\mathrm{B}} / \sigma_{\mathrm{A}}=0.414$. The diameter of the larger component of the mixture is set to $\sigma_{\mathrm{A}}=1$ in all simulations. All lengths are measured in units of $\sigma_{\mathrm{A}}$. The packing fraction of the mixture is defined as $\eta=\rho^{*} \pi\left(N_{\mathrm{A}} \sigma_{\mathrm{A}}^{2}+\right.$ $\left.N_{\mathrm{B}} \sigma_{\mathrm{B}}^{2}\right) / 4 N$, where $\rho^{*}=\rho \sigma_{\mathrm{A}}^{2}$ is the dimensionless number density. Following the approaches in the monodisperse studies, we use the following external potential: $V(\vec{r})=$ $V_{0} \sin (\vec{K} \cdot \vec{r})$ with $\vec{K}=(4 \pi / a, 0)$ and $a$ the lattice parameter of the $S_{1}(\mathrm{AB})$ lattice. The wavelength $\lambda=2 \pi /|\vec{K}|$ of the external potential is commensurate with the periodicity of the $S_{1}(\mathrm{AB})$ lattice.

These studies were carried out by Monte Carlo simulations in the $N V T$ ensemble. In order to facilitate equilibration additional nonlocal moves are attempted with particle displacements, which are integer multiples of the potential wavelength. The simulation box is set up to be slightly rectangular with $L_{x} / L_{y} \approx 1.178$ and $N=1848$ for these simulations. This choice allows not only a box-spanning square lattice to form, but also a box-spanning monodisperse triangular lattice of the larger component to develop. Periodic boundary conditions are employed in all simulations.

For the interaction of the mixture with the external field three cases can be distinguished: (I) only the smaller component interacts with the external field, (II) both components interact with the external field, (III) only the larger component interacts with the external field.

For low amplitudes, $V_{0}^{*}=V_{0} / k_{\mathrm{B}} T$, we found a novel phenomenon: in contrast to the monodisperse LIF scenario a laser induced de-mixing [4] sets in for all three cases. Laser induced de-mixing results in the coexistence of a small component enriched fluid with a droplet of a monodisperse crystalline structure formed by the larger component. The ordering mechanisms at work and the resulting monodisperse lattice structures differ for the three cases [3]. The ordering 


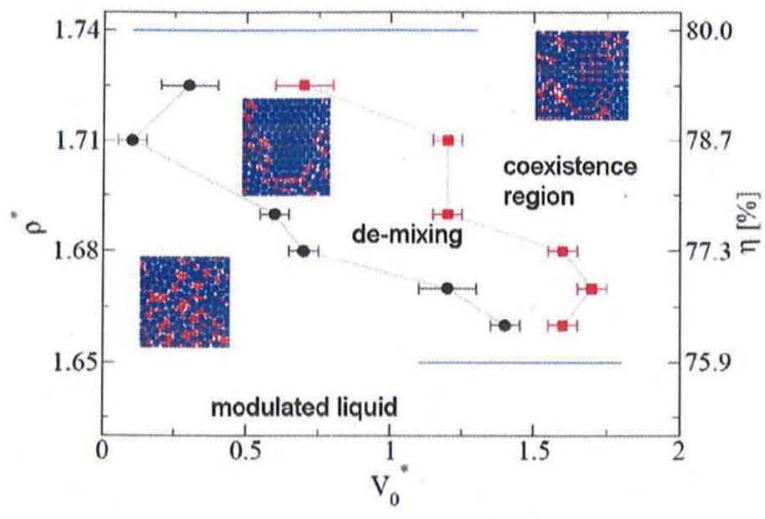

Figure 1. The laser induced de-mixing regime of the phase diagram for an equimolar binary mixture with diameter ratio $\sigma_{\mathrm{B}} / \sigma_{\mathrm{A}}=0.414$ exposed to an external periodic potential, commensurate with the $S_{1}(\mathrm{AB})$ square lattice for the case where only the smaller component of the mixture interacts with the external potential.

mechanisms stem from the attempt of the system to minimize its energy via an alignment of the components interacting with the external potential with the potential minima and the constraint of a fixed overall number density. While the coupling of only the smaller component to the external potential (case (I)) results in the coexistence of a monodisperse triangular lattice, cases (II) and (III), where the larger component interacts directly with the external potential, show a condensation of a monodisperse rhombic lattice.

There is no bulk de-mixing in the absence of a laser field due to the disparity in particle size in the mixture under study. The system exhibits two different coexistence regimes. One is the regime discussed in this paper, at weak external fields. Here one of the coexisting phases is a monodisperse ordered phase. This is in contrast to the second coexistence regime, which results at higher external field amplitudes. There the $50 \%$ mixture in its fluid phase coexists with a square lattice $\left(S_{1}(\mathrm{AB})\right)$, thus in this case no de-mixing occurs. The coexistence regime at weak external fields is called a de-mixing regime in order to emphasize this additional property of the coexistence regime at weak external fields. Its significance lies in the fact that an ordering in the larger component can be induced by coupling the smaller component to an external field.

Laser induced de-mixing offers an externally controllable way to manipulate the miscibility of the components of the mixture. In particular, for the scenario where only the smaller component interacts with the external potential, the regime of the phase diagram where de-mixing occurs can be obtained by analyzing the probability distribution of the shape factor $\zeta$ of the Voronoi cells. The shape factor is defined as the ratio of circumference to area of the cell and is a measure for the detection and characterization of structural changes in hard-disk fluids [15].

Here, in figure 1 we present the phase diagram for the laser induced de-mixing regime for this scenario. Above and below the horizontal blue line no laser induced de-mixing was observed. The figure depicts the phase boundaries between

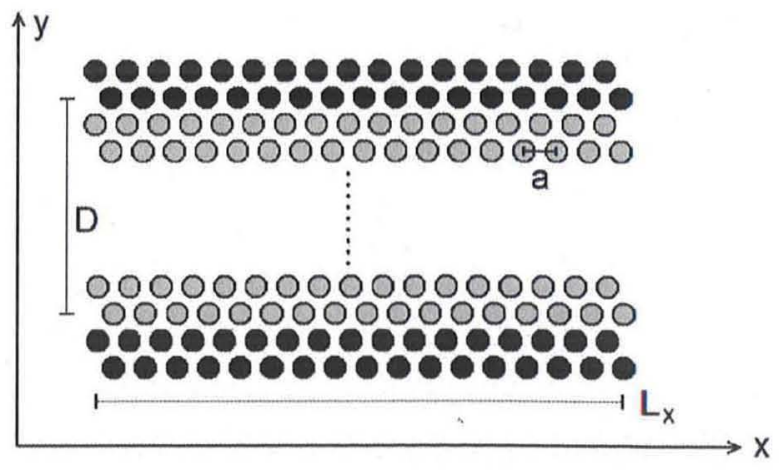

Figure 2. Sketch of the system geometry, showing the fixed wall particles (black spheres) and the mobile particles (gray spheres). The orientation of the coordinate axes is indicated, as well as the lattice spacing $a$ of the triangular lattice and the linear dimensions $L_{x}$ and $D$ of the system. When $D=n_{y} a \sqrt{3} / 2$ there is no misfit, $n_{y}$ rows of mobile particles (containing $n_{x}$ particles each, $L_{x}=n_{x} a$ ) occur at the approximately ideal distances relative to each other, while the choice $D=\left(n_{y}-\Delta\right) a \sqrt{3} / 2$ (with the misfit $\Delta$ ) implies uniaxial compression of the lattice. Typically we work with $n_{y}=30$ rows and $n_{x}=108$ (i.e., $N=3240$ mobile particles). Note that shear can be introduced in the system by moving the lower wall with velocity $v_{\text {shear }}$ in the $+x$-direction and the upper wall with the same velocity in the $-x$-direction.

the modulated liquid and the de-mixing regime as well as the boundary to a regime where square lattice structures and the de-mixed monodisperse structures compete.

\section{Two-dimensional colloidal crystals confined by one-dimensional corrugated 'walls': phase transitions and behavior under shear}

In this section we discuss simulation studies of a system of spherical colloids (which we model by point particles) interacting with each other via a short-ranged isotropic repulsive potential in a two-dimensional geometry and confined by corrugated repulsive walls (figure 2). Following our previous work [16-20], we define these walls in terms of two rows of particles fixed at the positions of a (perfectly ideal) rigid triangular lattice, which has a spacing commensurate with the lattice structure of the mobile particles (at the chosen density), see figure 2.

For our Monte Carlo simulations, we choose a generic model which is computationally efficient: a potential $V(r) \propto$ $r^{-12}$, but cut off at a finite range $r_{\mathrm{c}}$ (and shifted and smoothed, to avoid problems resulting from discontinuous forces). Specifically, we use

$$
V(r)=\left[\epsilon\left(\frac{\sigma}{r}\right)^{12}-\epsilon\left(\frac{\sigma}{r_{\mathrm{c}}}\right)^{12}\right] \cdot\left[\frac{\left(r-r_{\mathrm{c}}\right)^{4}}{h^{4}+\left(r-r_{\mathrm{c}}\right)^{4}}\right]
$$

with parameters $r_{\mathrm{c}}=2.5 \sigma, h=0.01 \sigma$ and the particle diameter $\sigma=1$ defining the length scale in our model. Similarly, $\epsilon=1$ defines the energy scale. At a density $\rho=N / V=1.05$ the melting transition then occurs at a temperature $T=T_{\mathrm{m}}=1.35[21]$ (note that $k_{\mathrm{B}}=1$ ). We are mostly interested in temperatures $T=1.0$ or lower here, i.e. deep in the crystalline phase. 


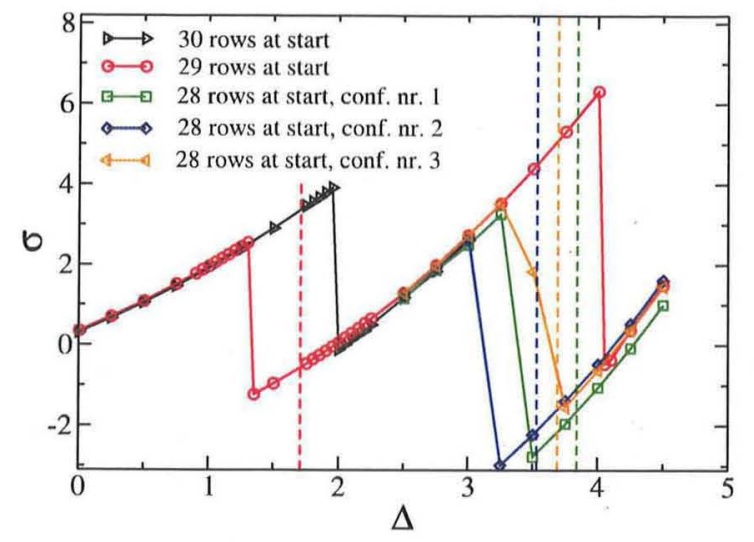

Figure 3. Stress anisotropy $\sigma=\sigma_{y y}-\sigma_{x x}$ plotted versus misfit $\Delta$, for a system of $N=3240$ mobile particles, and using different starting configurations, containing $n_{y}=30, n_{y}=29$ or $n_{y}=28$ rows. The vertical broken straight lines denote the positions of the equilibrium transitions, located by free energy methods. Note that the phase switch simulations showed that from the three candidate structures numbers $1-3$ with 28 rows structures numbers 1 and 3 are only metastable, while number 2 is the stable structure. All data are for $T=1.0$. Reproduced with permission from [22]. Copyright 2012 American Physical Society.

Already in our previous work it was shown that uniaxial compression of the system by reducing the available (two-dimensional) 'volume' via a reduction of the distance $D$ between the confining walls can introduce structural phase transitions in the system [18-20]. Increasing the misfit $\Delta$ (defined via $D(\Delta)=\left(n_{y}-\Delta\right) a \sqrt{3} / 2, D(0)=D_{0}, a$ being the lattice constant of the undistorted triangular lattice at the chosen density $\left.\rho=N /\left(D_{0} L_{x}\right)=1.05\right)$ one finds that the stress anisotropy $\sigma=\sigma_{y y}-\sigma_{x x}$ increases until at some value a jump discontinuity occurs, where $\sigma$ suddenly decreases, accompanied by a transition where the number of rows changes $\left(n_{y} \rightarrow\left(n_{y}-1\right)\right)$ while the total particle number $N$ in the system stays fixed.

This reduction in the number of layers means that the $n_{x}$ particles of the row that is removed are distributed in the remaining rows, leading to a smaller lattice spacing also in the $x$-direction. However, this smaller lattice spacing is incommensurate with the original lattice spacing $a$ in the rows of fixed particles representing the walls; this lack of commensurability leads to the formation of a 'soliton staircase' along the boundaries, and a nonuniform strain distribution throughout the system [18-20].

However, when one follows this transition in the opposite direction $\left(\left(n_{y}-1\right) \rightarrow n_{y}\right)$, one encounters a huge hysteresis (figure 3). Such a non-equilibrium behavior is not uncommon for discontinuous (first order) phase transitions, of course. Note that the situation gets worse when one compresses the system further, to observe the transition $\left(n_{y}-1\right) \rightarrow\left(n_{y}-2\right)$ (figure 3). Several 'candidate structures' for the phase with $n_{y}-2$ rows were found, but a priori it is unclear which is stable and which are only metastable.

To clarify the relative stability of such structures which are imperfectly crystallized and to locate the positions of the transitions in thermal equilibrium, a first principle method

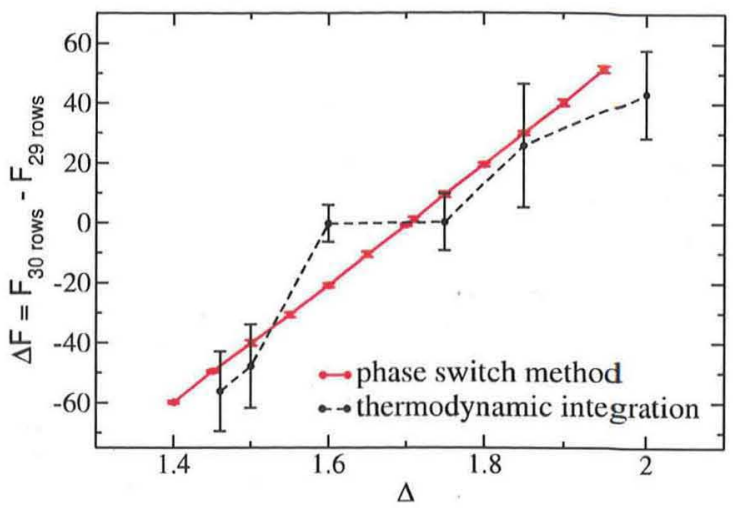

Figure 4. Free energy differences between the structures with $\left(n_{y}-1\right)=29$ and $n_{y}=30$ rows shown as functions of the misfit $\Delta$. The points with the large error bars are from the Schmid-Schilling thermodynamic integration method and the points falling on the straight line are from phase switch Monte Carlo simulations. Reproduced with permission from [22]. Copyright 2012 American Physical Society.

is to obtain the free energy difference between the various structures. There are several ways how this can be achieved. One approach that we have applied is to use the method of Schmid and Schilling [21] which separately estimates the absolute free energy of each structure in question. However, the obvious disadvantage of this method is that, for the transition from $n_{y}=30$ to $n_{y}=29$, for a misfit in the rangc $1.4 \leq \Delta \leq 2.0$, the total free energies $F_{\mathrm{I}}, F_{\mathrm{II}}$ of both phases vary from about 22000 to about 24000 , but their difference $\Delta F$ varies only from about -60 to +60 in that range. Thus reaching the necessary accuracy requires an enormous effort. We have found it much more efficient (see [22] for a more detailed explanation of technical aspects of this work) to employ the 'phase switch Monte Carlo method' [23, 24]. In this method, one directly carries out moves from states belonging to one phase to states belonging to the other phase, sampling the relative weights of both phases in the course of the simulation. Figure 4 shows, as an example, a plot of $\Delta F$ versus $\Delta$ for the $n_{y} \rightarrow\left(n_{y}-1\right)$ transition [22].

Having clarified which structures are stable and which are only metastable, it is of interest to explore the response of the system when the walls are sheared against each other (figure 5). One sees that the shear deformation 'melts' the crystal near the walls, where the velocity is nonzero, so that the particles flow with the wall. Note that unlike figures 3 and 4 these data are not obtained from Monte Carlo methods, of course, but rather using molecular dynamics methods (applying a Langevin thermostat to avoid the system steadily heating up; however, we do find at large velocities a nontrivial temperature profile $T(y)$ across the film [25]).

One should not attribute the shear melting of the crystal near the walls to the increase in local temperature (which is extracted from the velocity distribution of the particles); however, as figure 5 demonstrates, the range over which the velocity is distinctly nonzero in the system is largest for a wall velocity of $v=0.5$, for which the temperature enhancement near the walls is still small. That is, about five rows at 

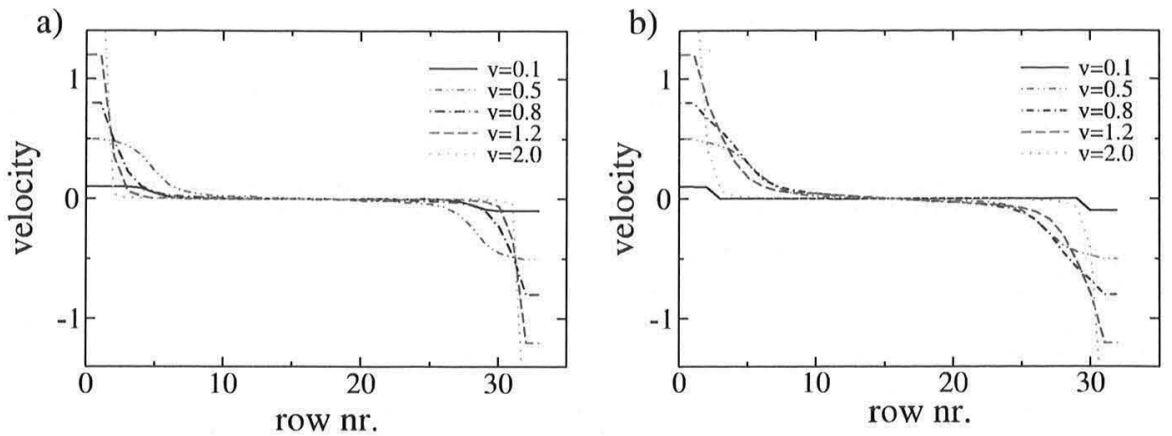

Figure 5. Velocity profile $v(y)$ at temperature $T=0.3$ and $n_{y}=30, \Delta=0$, i.e. a commensurate system with no misfit (a), and a system with $n_{y}=29$ rows at misfit $\Delta=2.2$ (b). Various choices of the wall velocity $v_{\text {shear }}$ are included, as indicated.

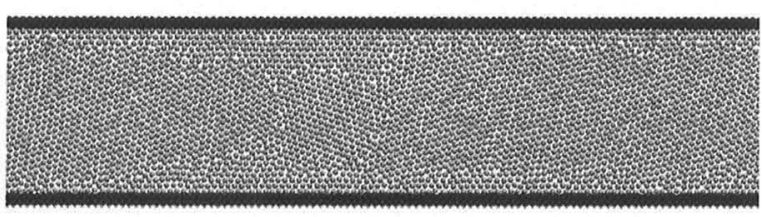

Figure 6. Snapshot of a configuration for a system at temperature $T=0.3$, misfit $\Delta=2.2, n_{y}=29$ rows and $v_{\text {shear }}=0.5$.

each wall are taking in this case fluid like configurations, as an examination of snapshot pictures reveals, see figure 6. It should also be noted that in the interior of the system the triangular crystal structure does not at all stay. intact, rather one finds large crystalline domains rotated by more or less large angles relative to the undeformed case. These domains are separated by grain boundaries. This structure is not completely static, in fact, for row numbers $n=10$ or $n=20$, which belong to this crystalline domain structure, one finds small but distinctly nonzero velocities (for wall velocity $v=0.5$, for instance), thus, the system exhibits plastic flow.

When the wall velocity increases beyond about $v=1.5$, however, the extent of flow that is caused in the system is much reduced: the wall particles already move too fast to be able to create a coherent motion in the boundary region of the crystalline strip. In fact, for $v \rightarrow \infty$ the situation corresponds to an effectively flat wall potential, which does not lead to any flow of the mobile particles. Also the local temperature increases only in the rows immediately adjacent to the walls (due to enhanced friction), while in the interior of the system $T(y)$ with increasing $v$ reaches a maximum and then decreases again.

Thus, shearing confined two-dimensional (and hence very soft) crystals causes very rich and diverse behavior (for a more detailed account of this work we refer to [25]).

\section{Transport in colloidal model systems}

\subsection{System, equilibrium and the effect of external fields in two-dimensional systems}

Non-equilibrium stationary states have received much attention recently in different areas of science ranging from

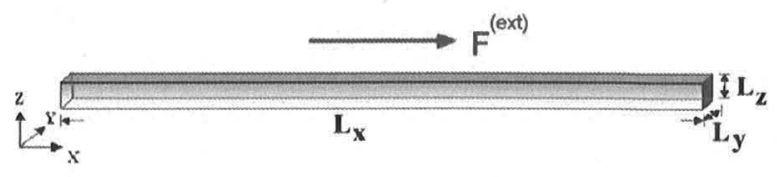

Figure 7. Three-dimensional microchannel with square cross section.

the analysis of pedestrian dynamics [26], to biologically relevant transport of ions in membranes [28], to lab-on-chip devices and microfluidics [27]. The behavior of colloidal systems [29] has been studied both in two-dimensional (2D) [30-32] and in three-dimensional (3D) systems [33-35]. Experiments for non-equilibrium structure formation have been carried out with oppositely charged colloids driven in opposite directions by an electric field [36] or in binary complex plasmas under microgravity conditions [37]. Such driven, diffusive systems serve as model systems for theoretical studies of non-equilibrium behavior [38].

Here we present results for the structure and dynamics of colloidal systems confined to two- and three-dimensional channels in equilibrium and under the action of external constant forces. Details of the model and methods have been presented elsewhere [39]. The channel geometry is sketched in figure 7 . The time evolution of the system is obtained by Brownian dynamics simulation, based on the overdamped Langevin equation. Hydrodynamic interactions as well as short time momentum relaxation of the particles are neglected. The appropriate stochastic position Langevin equation for this situation with friction constant $\xi$,

$$
\xi \frac{\mathrm{d} \mathbf{r}_{i}(t)}{\mathrm{d} t}=-\nabla_{\mathbf{r}_{i}} \sum_{i \neq j} \mathcal{V}_{i j}\left(r_{i j}\right)+\mathbf{F}_{i}^{(\mathrm{ext})}+\tilde{\mathbf{F}}_{i}(t),
$$

is time-integrated with a finite time step $\Delta t$ and the technique of Ermak [40]. The right-hand side includes the particle interaction as a sum over all forces acting on each particle, the constant driving force $\mathbf{F}_{i}^{(\text {ext) }}$ and the random forces $\tilde{\mathbf{F}}_{i}$ describing the random collisions of the solvent molecules with the $i$ th colloidal particle and modeled as a white noise term, representing a heat bath with temperature $T$. The 2D channels have a length of $L_{x}=800 \sigma$ and a width of $L_{y}=$ $10 \sigma$ containing 3200 particles corresponding to a density of 
$\rho=0.4 \sigma^{-2}$. The 3D channels typically have a quadratic square section of $L_{y}=L_{z}=5 \sigma$ and a length of $L_{x}=1000 \sigma$ containing about 10000 particles with a fixed particle density of $\rho=0.4 \sigma^{-3}$. The particles are confined within the channels by ideal hard walls. The equilibrium configuration in a closed channel is calculated starting from a random configuration and applying hard wall boundary conditions in all directions. The systems are typically equilibrated over $2 \times 10^{5}$ time steps. The time step $\Delta t=7.5 \times 10^{-5} t_{\mathrm{B}}$ is used, where $t_{\mathrm{B}}=$ $\xi \sigma^{2} / k_{\mathrm{B}} T$ is the time a particle needs in equilibrium to diffuse its own diameter $\sigma$. The friction constant $\xi$ is chosen to be $\xi=3 \pi \eta \sigma=4.288 \times 10^{-8} \mathrm{~N} \mathrm{~s} \mathrm{~m}^{-1}$, where $\eta=0.001 \mathrm{~Pa} \mathrm{~s}$ is the shear viscosity of water. The simulations are carried out at a constant temperature $T=295 \mathrm{~K}$. To study the influence of an external force, a constant driving force of $F^{(\mathrm{ext})}=$ $2.603 \times 10^{-15} \mathrm{~N}$ is applied in the longitudinal direction. The channel end is then realized by an open boundary condition. To keep the number density in the channel fixed a new particle is inserted within the first $10 \%$ of the channel at a random position avoiding overlaps every time a particle leaves the open end of the channel acting as a particle reservoir. This procedure seems to be appropriate to permit comparison with experimental studies [43,44], in which the channel is coupled to two reservoirs.

Due to a confinement, a classical 2D system forms a layered structure in equilibrium [41, 16, 43, 44], and the change in the number of layers due to the geometry of the confinement has been predicted using Langevin dynamic simulations [42]. The effect of the special boundary conditions in the $x$-direction has been analyzed in great detail for two-dimensional systems in [44]. A constant driving force in the $x$-direction and periodic boundary conditions would result in a layer formation for certain channel widths, similar to the equilibrium situation. The insertion of particles, which leave the channel end, at a randomly chosen place in the $10 \%$ region at the beginning of the channel results in a density gradient across the channel. At places along the channel, where the density has decreased sufficiently to energetically stabilize configurations with one layer less, the number of layers changes by -1 . This scenario is essentially the same for three-dimensional systems, except that the number of planes parallel to the 3D-channel walls gets reduced instead of the number of layers. In the case where the external forces are not acting on the particles in the 'reservoir regions' of the channel (the first and last $10 \%$ of the channel), interestingly a density increase results in the flow direction [44].

Even more interesting is the behavior of the system at barriers perpendicular to the channel and flow direction. A transparent, micron-sized colloidal particle, whose index of refraction is greater than that of the surrounding medium, can be trapped by a tightly focused laser beam. Extremely high gradients in the electric field occurring near the waist of the laser beam are associated with strong forces which drag the particle to the focal point of the laser beam. The strength of the optical force acting to restore the particle position to the trap center can be considered, in good approximation, to be harmonic. Therefore the force may be expressed by $F_{\text {opt }}(r)=$ $-k r$ for $r \leq R_{\text {trap }}$ and $F_{\text {opt }}(r)=0$ for $r>R_{\text {trap }}$, where $k$

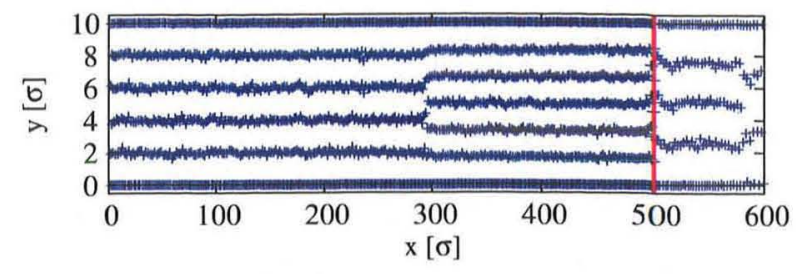

Figure 8. Full channel snapshot of a channel with a barrier of repulsive optical traps marked by the red line. The simulation parameters used are $L_{x}=800 \sigma, L_{y}=10 \sigma, n=0.3 \sigma^{-2}$, $B=0.5 \mathrm{mT}$, a driving force corresponding to a channel inclination angle of $\alpha=0.2^{\circ}$, and the trap parameters $\tilde{k}=-1, R_{\text {trap }}=0.75 \sigma$.

denotes the trap stiffness perpendicular to the direction of the laser beam and $R_{\text {trap }}$ is the interaction range of the trap. To study possible influences on the flow behavior simulations have been performed, where we additionally inserted the trap force $F_{\mathrm{opt}}$ into the overdamped Langevin equations which are evaluated numerically. These simulations model optical traps of different interaction ranges, in various geometrical arrangements ranging from fixed user-defined positions to fixed random positions, and barriers made out of traps transverse to the direction in which the particles are driven.

Figure 8 shows the stationary non-equilibrium situation of a barrier perpendicular to the particle driving direction being obtained from a BD simulation run. The particles are hindered from crossing the barrier. Thus, an increasing density gradient forms in front of the barrier leading to an increase of layers. At the barrier the local density shows a sharp non-continuous drop resulting in fewer layers. Further studies on the effect of barriers on the structure and dynamics of colloidal systems will be presented in [46] (same journal issue).

Following our studies in [43, 44, 39], we investigate the structural behavior of particles interacting via a hard-core Yukawa (YHC) potential in 2D and 3D microchannels by Brownian dynamics simulations. As a result of the layering effects due to the confinement by the channel walls, for different cross sections the particles can arrange into planes, parallel to the channel walls, for certain values of $L_{y}=L_{z}$, see figure 9 , or remain in a disordered configuration. Figure 10(a) shows the equilibrium configuration in a $3 \mathrm{D}$ channel with a quadratic square section of width $L_{y}=5 \sigma$ and a length of $1000 \sigma$. In equilibrium a loose structure with four planes forms which is stretched perpendicular to the confinement, see figure 10(b). The local lattice structure has been analyzed by local bond orientational order parameters [45]. In figure 9 we note that for $L_{y}=L_{z}=8.1 \sigma$, the number of planes is seven, systematically complementing findings of a previous study for other system sizes [39]. Due to the mismatch between the multiples of the lattice parameters and the channel width, the probability for lattice defects is minimal for ordered structures ( $5 \%$ for $L_{y}=5 \sigma$ (four planes), $14.2 \%$ for $L_{y}=6.3 \sigma$ (five planes) and $15 \%$ for $L_{y}=8.1 \sigma$ (seven planes)), compared to values for less ordered structures at slightly different channel widths ( $17 \%$ for $L_{y}=4.5 \sigma, 28.1 \%$ for $\left.L_{y}=5.5 \sigma\right)$, and the probability for a local fcc structure is roughly twice as large as that for local hcp structures. These results for the 
(a)
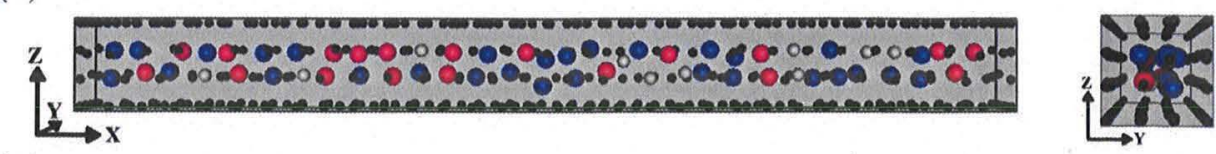

(b)
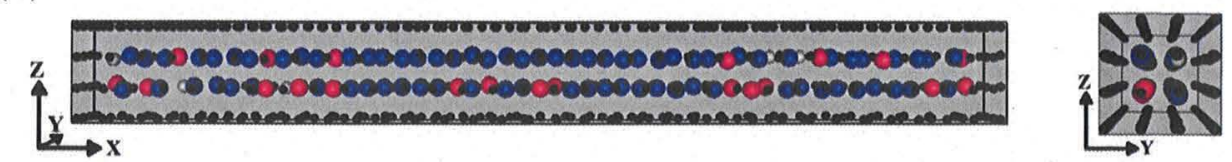

(c)

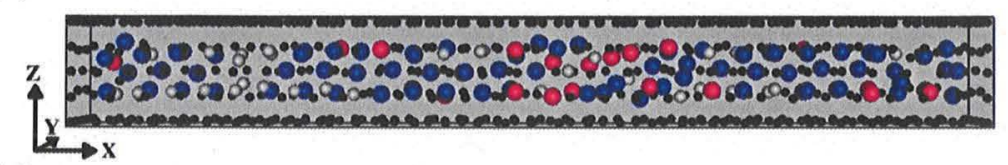

(d)
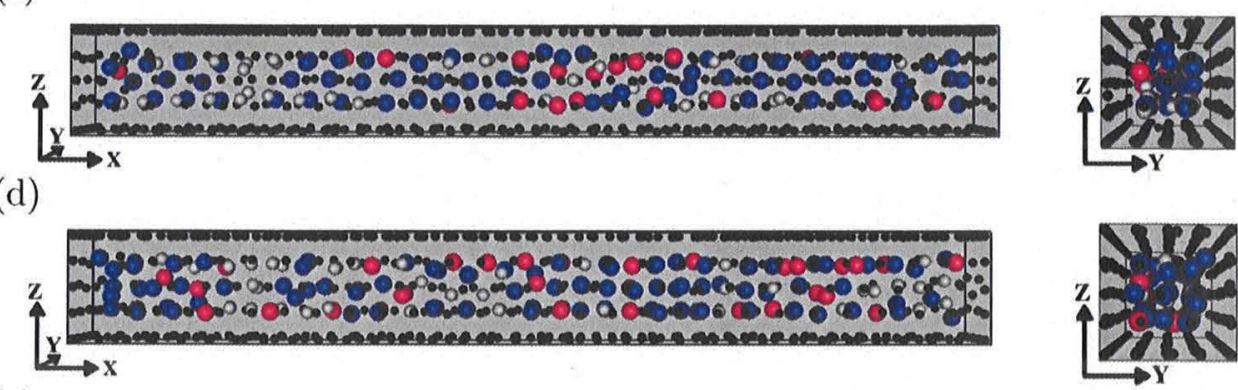

(e)
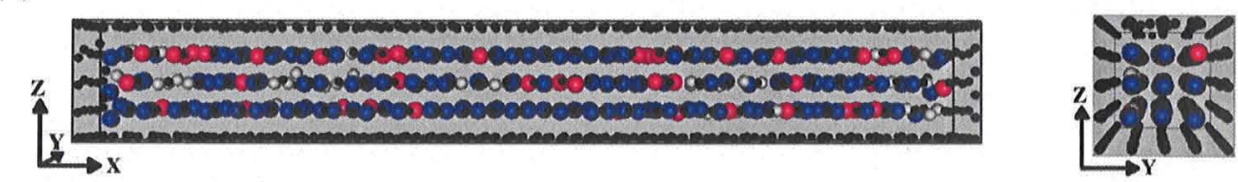

(f)
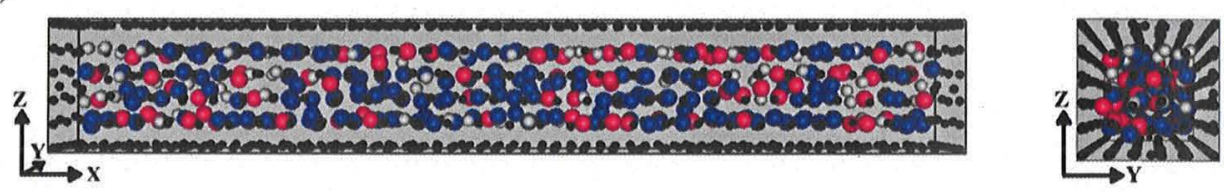

(g)
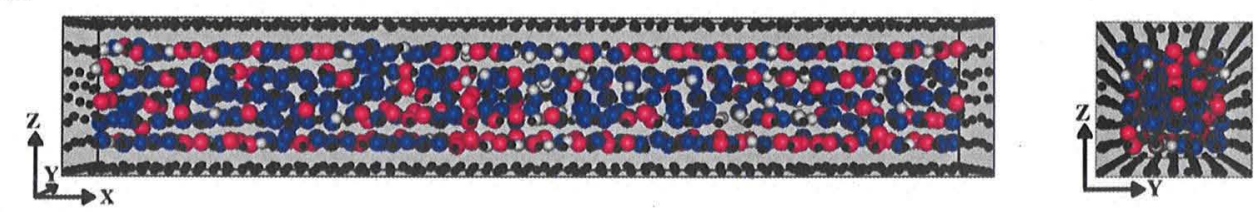

(h)
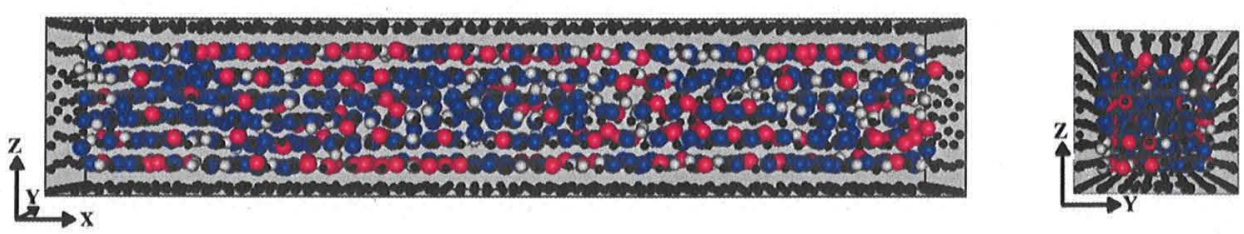

Figure 9. Crystal structures in microchannels (projected on the $y z$ plane) of different widths: (a) $L_{y}=4.5 \sigma$, (b) $L_{y}=5.0 \sigma$, (c) $L_{y}=5.5 \sigma$, (d) $L_{y}=5.7 \sigma$, (e) $L_{y}=6.3 \sigma$, (f) $L_{y}=6.9 \sigma$, (g) $L_{y}=8.1 \sigma$, (h) $L_{y}=9.3 \sigma$. Particles with fcc-symmetry are blue, with hep-symmetry red, defects are gray and boundary particles black.

defect probabilities complement our results in [44], in which the effect of the coupling constant on the defect densities was analyzed in two-dimensional systems interacting with a dipolar interaction. In the latter studies it was found that the defect densities increase with decreasing interaction strength, approaching fluid structures for small values, and that the presence of defects is closely related to the regions of layer reductions, whereas here we obtain greater and more detailed insight into the effect of the channel width on the defect probabilities.

\subsection{Transport behavior of colloids in $3 D$ microchannels}

Now we address the transport behavior of colloids confined in $3 \mathrm{D}$ microchannels. The colloids are driven by the application of an external driving force. A reduction of the number of 

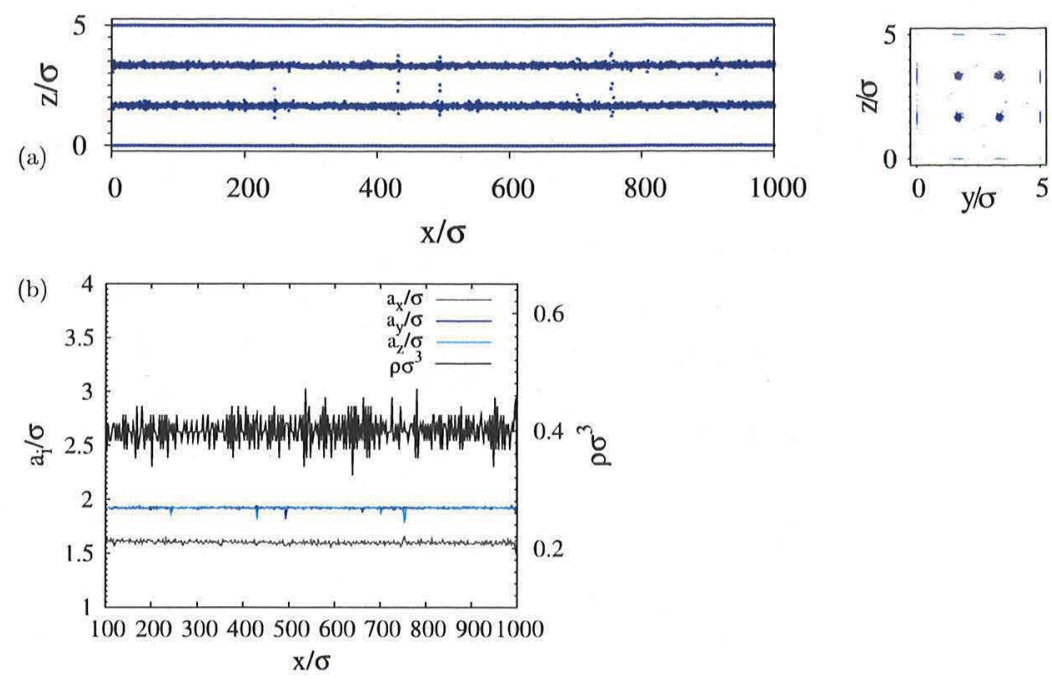

Figure 10. (a) Configurations in equilibrium. On the right side all particle coordinates are projected on the $y z$ plane, on the left side projections on the $x z$ plane are shown. The distances in the $z$-direction are stretched by a factor of 16. (b) Local density and lattice constants $a_{x}, a_{y}$ and $a_{z}$ in the channel in equilibrium.
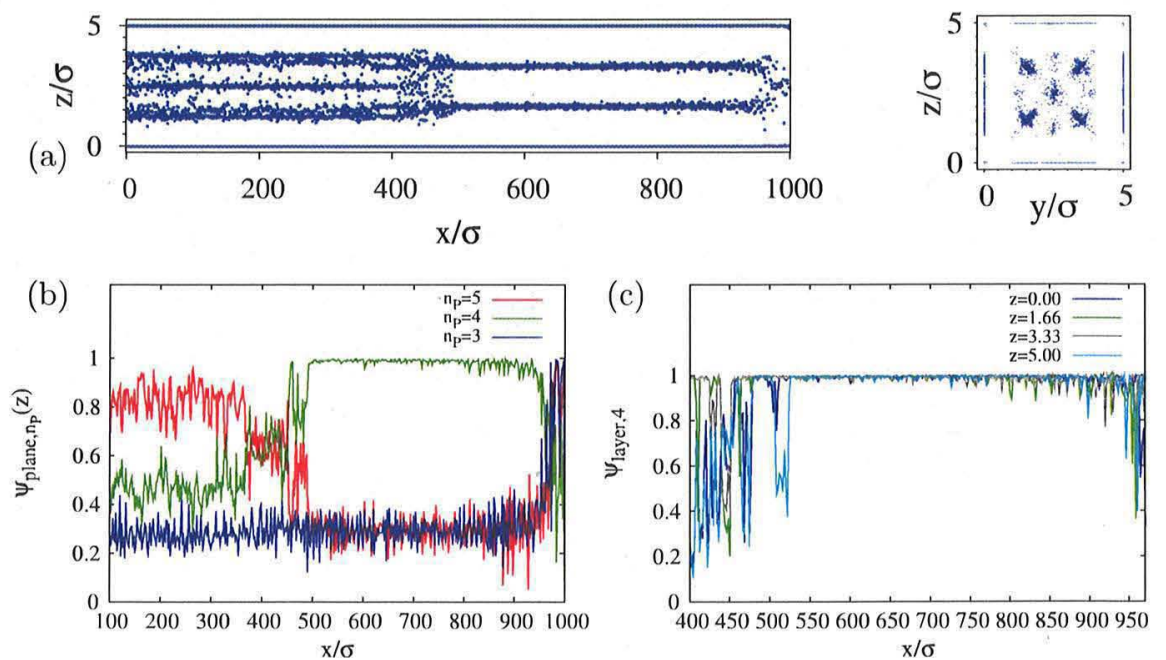

Figure 11. (a) Configurations in a non-equilibrium stationary state after $10^{6}$ time steps. On the right side all particle coordinates are projected on the $y z$ plane, on the left side projections on the $x z$ plane are shown. The distances in the $z$-direction are stretched by a factor of 16. (b) Plane order parameter for numbers of planes $n_{\mathrm{P}}=3, n_{\mathrm{P}}=4$ and $n_{\mathrm{P}}=5$ perpendicular to the $z$-direction. (c) Layer order parameter for the number of lanes $n_{1}=4$ for four planes in the $x y$-direction.

planes in the direction of the force can be seen in figure 11(a). This phenomenon is analogous to the layer reduction observed in the previous studies in 2D and 3D microchannels [43, 44, 39]. After $10^{6}$ time steps the system reaches a stationary non-equilibrium, in which the position at which a reduction of the number of planes occurs, see figure 11(a), is stable over long simulation times (quasi-stationary), and the particles move across this position. In the stationary non-equilibrium a density gradient forms along the complete length of the channel causing the change of the number of planes and therefore leading to a change of the lattice constants. While the density reduction is smooth and quasi-continuous within statistical scatter throughout the entire channel, the local lattice constants show a non-continuous behavior at the plane reduction regions. At the left end of the channel the lattice constant $a_{x}$ is larger then $a_{y}$ and $a_{z}$ and the lattice is compressed perpendicular to the confinement. At the end of the transition area $a_{x}$ is smaller than $a_{y}$ and $a_{z}$. While the latter two remain constant until the next transition point, $a_{x}$ increases monotonically. This behavior suggests that stretching of the lattice in the flow direction leads to an instability causing the reduction of the number of planes. The change in the number of planes is clearly visible from the plane order parameter [39], shown in figure 11(b), and defined as

$$
\Psi_{\text {plane }}\left(n_{\mathrm{p}}\right)=\left|\frac{1}{n_{\text {bin }}} \sum_{j=1}^{n_{\text {bin }}} \exp \left(\mathrm{i}\left(2 \pi\left(n_{\mathrm{p}}-1\right) / L_{\mathrm{p}}\right) p_{j}\right)\right|,
$$


$L_{y}$

2.3

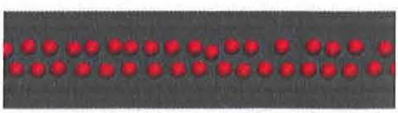

3.8

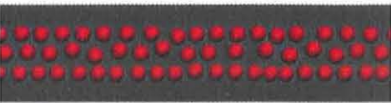

5.5

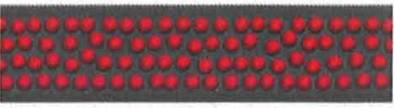

7.1

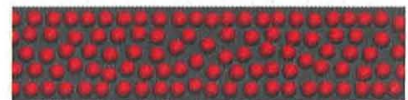

$L_{y}$

3.2

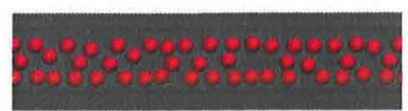

4.6

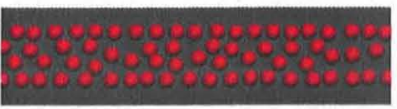

6.1

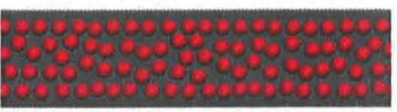

8.0

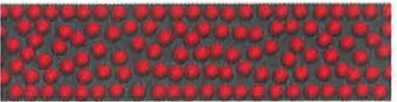

Figure 12. Snapshots of different channel configurations with maximal order (left) and minimal order (right). The diffusion in ordered systems is suppressed whereas the diffusion in disordered systems is enhanced [47].

(a)

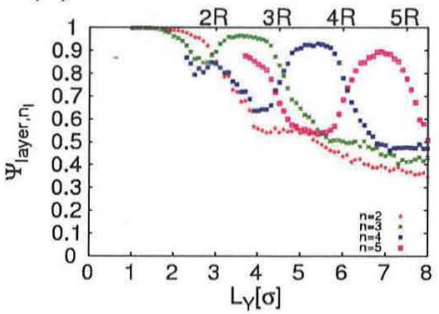

(b)

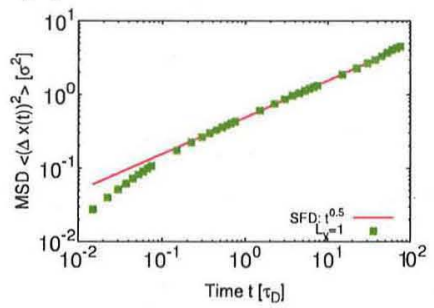

(c)

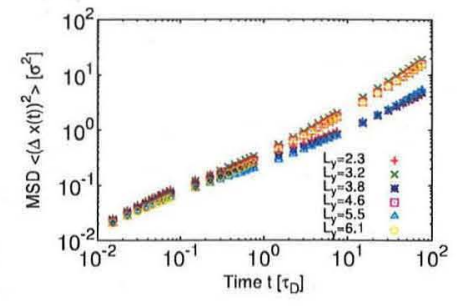

Figure 13. (a) The layer order parameters $\Psi_{\text {layer, } n_{1}}$ as a function of channel width for $n_{1}=2,3,4$ and 5 layers. (b), (c) The mean square displacements as a function of time in a $\log -\log$ plot for different channel widths [47].

with $p_{j} \in\left\{x_{j}, y_{j}\right\}$ and $L_{\mathrm{p}} \in\left\{L_{y}, L_{z}\right\}$. Here, the channel with cross section $L_{y} \times L_{z}$ is subdivided in the $x$-direction into regions containing $n_{\text {bin }}$ particles, and $\Psi_{\text {plane }}\left(n_{\mathrm{p}}\right)=1$ for configurations with $n_{\mathrm{p}}$ equidistant planes in the $y$ - or $z$-direction. In figure 11(c) we analyze the structural changes in greater detail by the layer order parameter [43, 44, 39], defined as

$$
\Psi_{\text {layer }}\left(n_{1}\right)=\left|\frac{1}{n_{\text {bin }}} \sum_{j=1}^{n_{\text {bin }}} \exp \left(\mathrm{i}\left(2 \pi\left(n_{1}-1\right) / L_{y}\right) y_{j}\right)\right| .
$$

Here, the (2D) channel of width $L_{y}$ at fixed $z$ is subdivided in the $x$-direction into regions containing $n_{\text {bin }}$ particles, and $\Psi_{\text {layer }}\left(n_{1}\right)=1$ for configurations with $n_{1}$ equidistant layers in the $y$-direction.

We note that in the part of the channel where four planes are stabilized, the number of layers in two-dimensional slices parallel to the $x y$ plane is four at z-positions in the channel, where the planes are located (at the walls, $z=0$ and $z=5 \sigma$, and at $z=1.66 \sigma$ and $z=3.33 \sigma$ ), proving good $2 \mathrm{D}$ as well as $3 \mathrm{D}$ ordering for this channel cross section $\left(L_{y}=L_{z}=5 \sigma\right)$.

\subsection{Diffusion of model colloids in $2 D$ microchannels}

The effects of confined geometry and of pinning sites on the diffusion of particles have been studied here and in section 5 (experiment). In both situations, a subdiffusive behavior has been found for certain time regions. Here, we have studied the effect of the layering in a two-dimensional colloidal system on the diffusion behavior. At a constant dimensionless interaction strength and constant density, layered systems and more disordered systems alternate with increasing channel width $L_{Y}$, as figure 12 shows. This type of layering order can also be characterized by a layer order parameter $\Psi_{\text {layer }, n_{1}}[43$, 44,39 ], which is near 1 if the particles are structured in $n_{1}$ equidistant layers and otherwise much smaller (figure 13(a)). This layering has an important influence on the diffusion, which can be characterized by the mean square displacement (MSD),

$$
\left\langle\Delta \vec{r}^{2}(t)\right\rangle=\frac{1}{N} \sum_{i=1}^{N}\left|\vec{r}_{i}(t)-\vec{r}_{i}(0)\right|^{2},
$$

where the sum runs over all particles $N$. For diffusion in the $x$-direction only the $x$ coordinates are considered in $\left\langle(\Delta x(t))^{2}\right\rangle$.

Starting from the well known case of single file diffusion [48] at channel width $L_{Y}=1$ (figure 13(b)) with a long time evolution of the MSD, $\left\langle(\Delta x(t))^{2}\right\rangle \propto t^{0.5}$, we studied the dependence of the MSD on the width of the channels. The MSD for wider channels has a non-monotonic dependence on the width $L_{Y}$. This non-monotonic behavior is induced by the layering. Figure 13(c) shows the MSD for the channels with maximal order $\left(L_{Y}=2.3 ; 3.8 ; 5.5\right)$ and with minimal 


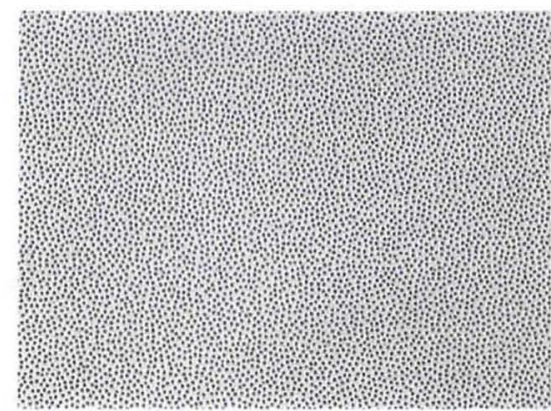

(a)

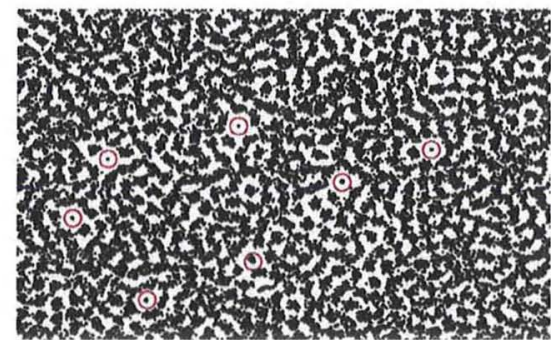

(c)

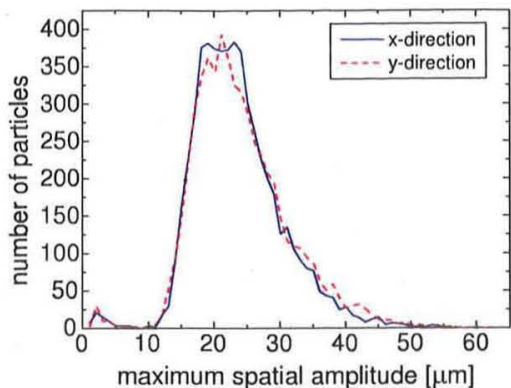

(b)

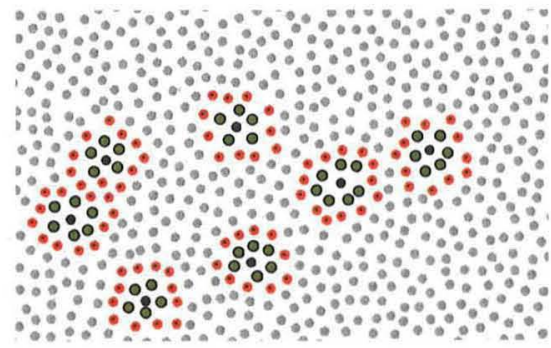

(d)

Figure 14. (a) Snapshot of the colloidal ensemble at the first time step. (b) Distribution of the maximum spatial amplitude of the single particle positions during $5000 \mathrm{~s}$. Particles are defined as pinned if their amplitude is below $9 \mu \mathrm{m}$. (c) Trajectories after $5000 \mathrm{~s}$ in a sub-window of the ensemble. The pinned particles are highlighted with circles. (d) The same sub-window as in (c), division of the ensemble into subsystems: pinned sites (black), their nearest (green) and next nearest neighbors (orange), and the rest of the particles (gray).

order $\left(L_{Y}=3.2 ; 4.6 ; 6.1\right)$. The diffusion has a maximum for disordered channels and a minimum for channels with layering. In all cases a subdiffusive exponent is found in certain intermediate time regions and at late times the standard diffusion exponent is found. This work is still in progress [47].

\section{Dynamics of a two-dimensional colloidal liquid with pinning}

A special way to confine particles is a set of pinning sites distributed randomly within a two-dimensional plane. This $(2 D-\epsilon)$-dimensional system is less artificial than it may appear. The thermodynamics of two-dimensional crystallization in random potentials is of particular interest since Nelson discovered the possibility of a melting transition due to quenched random impurities [49]. Chudnovsky and Serota generalized this scenario to substrates with random force fields [50, 51] and Cha and Fertig found a disorder induced melting at zero temperature [52]. In this context, we want to study the effect of confinement due to random pinning on the dynamics of a two-dimensional colloidal system.

Our ensemble consists of epoxy coated, superparamagnetic polystyrene beads with a size of $4.5 \mu \mathrm{m}$. Diluted in water and confined in a cylindrical glass cell with a radius of $5 \mathrm{~mm}$ and a height of $1.5 \mathrm{~mm}$, the particles sediment down to the bottom glass plate due to their higher mass density. We obtain one monolayer consisting of $\sim 10^{5}$ particles of which approximately $1 \%$ are pinned to the glass substrate. The pinning occurs due to a linking of epoxy polymers to the glass surface. The system is fixed at room temperature and inclined by precise micrometer motors to ensure a perfect horizontal alignment of the layer. The beads are doped with nanometer-sized $\mathrm{Fe}_{2} \mathrm{O}_{3} / \mathrm{Fe}_{3} \mathrm{O}_{4}$ domains which cause superparamagnetic behavior with a high magnetic susceptibility $\chi$ per bead. An external magnetic field $H$ perpendicular to the monolayer aligns the dipoles with particle density $n$ and allows us to tune the interaction strength between the beads, indicated by the dimensionless parameter

$$
\Gamma=\frac{(\pi n)^{3 / 2} \chi^{2} H^{2}}{4 \pi k_{\mathrm{B}} T} .
$$

$\Gamma$ is the ratio of magnetic to thermal energy which is a measure of the inverse system temperature $1 / T$. A window of $1 \mathrm{~mm}^{2}$ consisting of a few thousand beads is monitored permanently by video microscopy and the particles are tracked for a few hours in steps of a few seconds. This allows us to generate precise static and dynamic correlation functions in the long time limit.

We prepared the colloidal system at an interaction strength of $\Gamma \approx 37.0$. At this value, the system is in the isotropic liquid phase [53]. Figure 14(a) shows a snapshot of our ensemble which consists of approximately 5800 particles. Figure 14(b) shows the distribution of the maximum spatial amplitude of the particles in the $x$ - and $y$-directions. The amplitude is calculated by the magnitude of the difference between the minimum and maximum particle positions during $5000 \mathrm{~s}$. One can see that most particles cover the region with amplitudes between 15 and $35 \mu \mathrm{m}$, separated from particles with smaller amplitudes. We define particles as pinned if their maximum spatial amplitude is less than $9 \mu \mathrm{m}$ in the $x$ - and in 
$y$-directions. This threshold is also geometrically reasonable because it is twice the particle diameter and exactly the displacement a particle needs to get out of the way of another one. Figure 14(c) shows a trajectory plot of a sub-window of the colloidal system for $5000 \mathrm{~s}$. The pinned particles are highlighted with circles and one can see that these particles are clearly restricted in their movement.

We are now interested in the effect of the pinned particles on the dynamics of the rest of the ensemble. This effect should be more crucial for particles near the pinned ones than for the bulk. Therefore we subdivide the system into nearest neighbors and next nearest neighbors of the pinned particles, and the rest of the system. This classification can be seen in figure 14(d), where the pinned particles are colored black, nearest neighbors green, next nearest neighbors orange and the rest of the ensemble gray. To study the dynamics of the different subsystems, we look at the characteristics of the mean square displacement, see equation (5). The result is shown in figure 15 for the different subsystems, where the square root of the mean square displacement (sqrt-MSD) is plotted. One can see that there is a significant difference in the dynamics for the nearest neighbors observable, compared to freely moving particles in the bulk. This is caused by their partial confinement due to the pinned particles. Here, the nearest neighbors are those determined in the first time step since in MSD positions are compared to the initial ones. There is even a slight drop in the sqrt-MSD for the next nearest neighbors in the second shell, which indicates that the restricted dynamics due to the confinement is transferred over 'free' particles. For the pinned particles the sqrt-MSD is bounded at long times. It is interesting to note that the timescale where deviations between first shell and bulk particles are visible $(\sim 250 \mathrm{~s})$ is comparable to a few Brownian timescales. After about $3000 \mathrm{~s}$ the particles have moved more than a typical particle distance and are typically not nearest neighbors to the initial ones. This marks the range where motion is diffusive again for all but the pinned particles.

The dynamics of the nearest neighbors is clearly constricted and this effect is even seen for the next nearest neighbors. Approaching a more ordered phase, this might have a crucial effect on transition temperatures, the character and the microscopic mechanism of the phase transition. Not only might fluctuations on all length scales be suppressed which are essential for the KTHNY melting scenario [54-56], but a system with random pinning might also show similar thermodynamics to systems with short or long scale disordered substrates [57] or in confined geometries [16].

\section{Concluding remarks}

This work contains parts reviewing previous work by the authors as well as new, unpublished results. Among the latter are detailed studies of the phase boundaries of the de-mixing regime in binary systems in external light fields, configurations for shear induced effects at structured walls, studies on the effect of confinement on certain structures and defect densities in three-dimensional systems, the effect

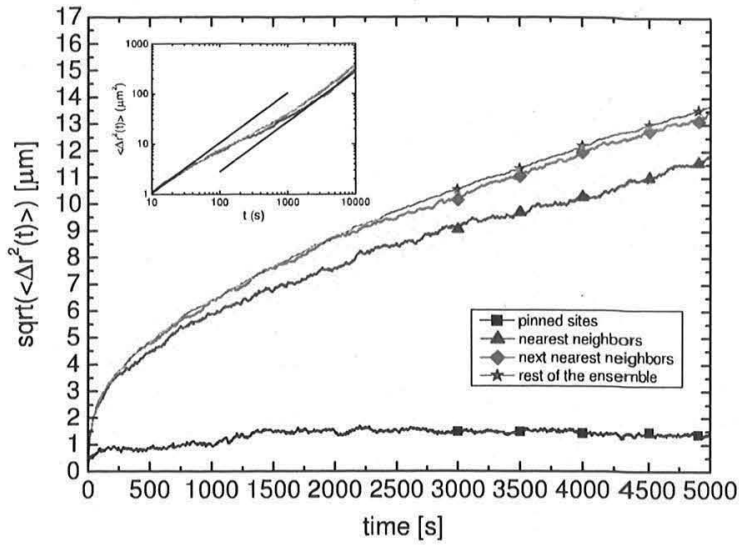

Figure 15. Sqrt-MSD for the different subsystems. The nearest neighbors of the pinned particles show a significant difference in their dynamics with respect to the rest of the ensemble, which is even seen for the next nearest neighbors. The inset shows the MSD in log-log scale. After about $3000 \mathrm{~s}$ the motion is diffusive again as indicated by the two lines with slope unity.

of confinement and barriers on two-dimensional flow and diffusion, and the effect of pinning sites on the diffusion.

The majority of our studies were carried out in two-dimensional systems. Sections 2 and 3 show new details on the effects of external light and shear fields on the structural properties of colloidal systems: the phase boundaries of an interesting light induced de-mixing effect as well as shear induced structural deformations near walls. The effect of the confinement on the dynamics of such systems was studied in greater detail in section 3 as well as in section 4, where particles were exposed to external fields in addition to the wall confinement. The first particle layers close to the walls are of particular interest in section 4 , where interesting layering effects were found for certain well chosen wall distances, resulting in a small number of layers. In section 3 complementary studies were carried out with the focus on larger layer numbers and the effect of shear on the ordering in the first layers close to the confining walls. Section 4 compares the effects known for two-dimensional systems with those of three-dimensional systems and presents more insight into the latter. In sections 4 and 5 finally the diffusion of particles was analyzed in greater detail. In section 5, the effect of pinning sites was studied by experimental methods, and an interesting subdiffusive behavior was found. In section 4 the formation of layers hinders the free diffusion of the particles resulting in a subdiffusive behavior as well. In both systems, this behavior is only found at intermediate timescales.

In this paper, we have presented model calculations on colloidal systems in strictly two-dimensional geometry, considering also the effect of confinement to quasi-onedimensional slit pores or channels, and dealt also with transport in quasi-one-dimensional channels having a square cross section. One of the perturbations of the ordering that we considered was the uniaxial compression of colloidal crystals (that in the ideal case form a perfect triangular lattice structure) by corrugated walls, that (in the case without misfit) are commensurate with the crystal structure. We have studied 
the phase transitions that occur in the number of layers that take place with increasing misfit ( $n$ layers $\rightarrow n-1$ layers $\rightarrow n-2$ layers). We studied the increasing disorder that this compression (together with the resulting incommensurability at the walls) causes. Since these transitions display huge hysteresis, and also in some cases several different structures occur with comparable degree of disorder, it has not been clear which of these structures is the stable one, and which are only metastable. To answer such questions, we found it useful to develop methods from which the free energy differences between different (partially ordered) structures can be inferred. Comparing results obtained from either the Schmid-Schilling method or the 'phase switch Monte Carlo' method of Wilding, we found that both methods give mutually compatible results, but the phase switch Monte Carlo method gives much more accurate results even with less numerical effort. We believe that this method should also be useful for other cases where in colloidal systems several (partially or perfectly) ordered phases compete with each other. Another interesting perturbation occurs if the two confining walls are not used to compress the system in the direction perpendicular to the walls, but rather to create a shear deformation, moving both walls along the strip, but in antiparallel directions. This causes a kind of 'melting' of a few layers of the crystal along the wall. The behavior of such a deformed crystal is reminiscent of 'shear banding', familiar from other soft matter systems under flow; however, the investigation of the details of this interesting behavior must be left to future work.

A successful route to the controlled structuring of binary mixtures is exposure of the system to a modulated external field. Weak external fields allow a controlled tuning of the miscibility of the mixture. The ordering mechanisms resulting in a laser induced de-mixing (LID) in this regime depend on the details of the coupling of the components of the mixture to the modulating field $[3,4]$. Here we presented the phase diagram of the laser induced de-mixing regime for the case where only the smaller component of the mixture interacts with the external potential.

In addition, a structural analysis has been performed in a colloidal model system with dipolar or YHC particle interactions in 2D and 3D microchannels. We have reported on a variety of ordering and transport phenomena induced by the confinement of the motion of the particles by parallel walls and by the application of a constant driving force along the channel. The structural properties show oscillations upon increasing the width of the channel with a period of the effective boundary plane distance. Due to the mismatch between the multiples of the lattice parameters and the channel width, the probability for lattice defects is minimal for ordered structures compared to the values for less ordered structures, and the probability for a local fcc structure is roughly twice as large as for local hcp structures. The number of planes can be reduced by applying an external driving force. In the stationary non-equilibrium the particles flow over the reduction zone which stays at a constant position. For small driving forces, where the particles are not in the regime of plug flow, the particles arrange themselves into a different number of planes, and in these planes parallel to the channel walls a good layer ordering is found in addition, showing good ordering in 3D as well as in 2D. The effect of the layering in 2D systems on the diffusion behavior is briefly discussed.

In an experimental system we have shown that point like defects can affect the mobility of particles locally. The mean squared displacement of particles which are nearest neighbors of particles which are immobile due to pinning to the substrate is significantly reduced in a 2D fluid. The effect is even visible in the second shell of pinned particles. This locally reduced mobility caused by pinning centers may affect the nature of phase transition in 2D experimental systems where critical fluctuations play an important role.

\section{Acknowledgments}

The authors thank the SFB-TR6 for support and the NIC for computer time. Some of us (DW, KB, PV) also thank Surajit Sengupta and Nigel B Wilding for their valuable collaboration on simulations of colloidal crystals confined by corrugated walls, and they also thank T Schilling, F Schmid and I Snook for helpful discussions.

\section{References}

[1] Franzrahe K, Nielaba P and Sengupta S 2010 Phys. Rev. E 82016112

[2] Franzrahe K, Keim P, Maret G, Nielaba P and Sengupta S 2008 Phys. Rev. E 78026106

[3] Franzrahe K and Nielaba P 2009 Phys. Rev. E 79051505

[4] Franzrahe K and Nielaba P 2007 Phys. Rev. E 76061503

[5] Bürzle F and Nielaba P 2007 Phys. Rev. E 76051112

[6] Strepp W, Sengupta S and Nielaba P 2001 Phys. Rev. E 63046106

[7] Strepp W, Sengupta S and Nielaba P 2002 Phys. Rev. E 66056109

[8] Chowdhury A and Ackerson B J 1985 Phys. Rev. Lett. 55833

[9] Wei Q H, Bechinger C, Rudhardt D and Leiderer P 1998 Phys. Rev. Lett. 812606

[10] Chakrabarti J, Krishnamurthy H R, Sood A K and Sengupta S 1995 Phys. Rev. Lett. 752232

[11] Chaudhuri P, Das C, Dasgupta C, Krishnamurthy H R and Sood A K 2005 Phys. Rev. E 72061404

[12] Chakrabarti J, Krishnamurthy H R and Sood A K 1994 Phys. Rev. Lett. 732923

[13] Frey E, Nelson D R and Radzihovsky L 1999 Phys. Rev. Lett. 832977

[14] Radzihovsky L, Frey E and Nelson D R 2001 Phys. Rev. E 63031503

[15] Moucka F and Nezbeda I 2005 Phys. Rev. Lett. 94040601

[16] Ricci A, Nielaba P, Sengupta S and Binder K 2006 Phys. Rev. E 74010404

[17] Ricci A, Nielaba P, Sengupta S and Binder K 2007 Phys. Rev. E 75011405

[18] Chui Y-H, Sengupta S and Binder K 2008 Europhys. Lett. 8358004

[19] Chui Y-H, Sengupta S, Snook I K and Binder K 2010 J. Chem. Phys. 132074701

[20] Chui Y-H, Sengupta S, Snook I K and Binder K 2010 Phys. Rev. E 81020403

[21] Schilling T and Schmid F 2009 J. Chem. Phys. 131231102

[22] Wilms D, Wilding N B and Binder K 2012 Phys. Rev. E 85056703

[23] Bruce A D, Wilding N B and Ackland G J 1997 Phys. Rev. Lett. 793002 
[24] Wilding N B and Bruce A D 2000 Phys. Rev. Lett. 855138

[25] Wilms D, Virnau P, Sengupta S and Binder K 2012 Phys. Rev. E 85061406

[26] Helbing D, Molnar P, Farkas I and Bolay K 2001 Environ. Plan. B 28361

[27] Squires T M and Quake S R 2005 Rev. Mod. Phys. 77977

[28] Roth R and Gillespie D 2005 Phys. Rev. Lett. 95247801

[29] Löwen H 2001 J. Phys.: Condens. Matter 13 R415

[30] Rex M, Löwen H and Likos C N 2005 Phys. Rev. E 72021404

[31] Rex M and Löwen H 2007 Phys. Rev. E 75051402

[32] Rex M and Löwen H 2008 Eur. Phys. J. E 26143

[33] Chakrabarti J, Dzubiella J and Löwen H 2004 Phys. Rev. E 70012401

[34] Dzubiella J and Löwen H 2002 J. Phys.: Condens. Matter 149383

[35] Chakrabarti J, Dzubiella J and Löwen H 2003 Europhys. Lett. 61415

[36] Leunissen M E, Christova C G, Hynninen A P, Royall C P, Campbell A I, Imhof A, Dijkstra M, v Roij R and v Blaaderen A 2005 Nature 437235

[37] Sütterlin K R et al 2009 Phys. Rev. Lett. 102085003

[38] Schmittmann B and Zia R K P 1995 Phase Transition and Critical Phenomena vol 17 (New York: Academic)

[39] Schwierz N and Nielaba P 2010 Phys. Rev. E 82031401
[40] Ermak D L 1975 J. Chem. Phys. 624189

[41] Haghgooie R and Doyle P S 2004 Phys. Rev. E 70061408

[42] Piacente G and Peeters F M 2005 Phys. Rev. B 72205208

[43] Köppl M, Henseler P, Erbe A, Nielaba P and Leiderer P 2006 Phys. Rev. Lett. 97208302

[44] Henseler P, Erbe A, Köppl M, Leiderer P and Nielaba P 2010 Phys. Rev. E 81041402

[45] Steinhardt P J, Nelson D R and Ronchetti M 1983 Phys. Rev. B 28784

[46] Kreuter C, Siems U, Henseler P, Nielaba P, Leiderer P and Erbe A 2012 J. Phys.: Condens. Matter 24464120

[47] Siems U Dissertation (U Konstanz) in preparation

[48] Wei Q H, Bechinger C and Leiderer P 2000 Science 287625

[49] Nelson D R 1983 Phys. Rev. B 272902

[50] Chudnovsky E M 1986 Phys. Rev. B 33245

[51] Serota R A 1986 Phys. Rev. B 333403

[52] Cha M-C and Fertig H A 1995 Phys. Rev. Lett. 744867

[53] Keim P, Maret G and von Grünberg H H 2007 Phys. Rev. E 75031402

[54] Kosterlitz J M and Thouless D J 1973 J. Phys. C: Solid State Phys. C 61181

[55] Halperin B I and Nelson D R 1978 Phys. Rev. Lett. 41519

[56] Young A P 1979 Phys. Rev. B 191855

[57] Carpentier D and Le Doussal P 1998 Phys. Rev. Lett. 811881 